\title{
IIMA

\section{Stability Behaviour in Functional Differential Equations of the Neutral Type}

\author{
Ali Fuat Yeniçerioğlu ${ }^{1 *}$, Cüneyt Yazıcı ${ }^{1}$ and Vildan Yazıcı ${ }^{2}$ \\ ${ }^{1}$ Kocaeli University, Faculty of Education, Kocaeli, 41380, Turkey \\ ${ }^{2}$ Kocaeli University, Faculty of Arts and Sciences, Kocaeli, 41380, Turkey
}

\section{Article Info}

Keywords: Asymptotic behavior, Functional differential equation, Neutral, Characteristic equation, Stability 2010 AMS: $34 K 06,34 K 20,34 K 25$, $34 K 38,34 K 40$.

Received: 30 November 2020

Accepted: 1 March 2021

Available online: xxxx March 2021

\begin{abstract}
In this study, we examine the behavior of solutions of the neutral functional differential equations. Using a suitable real root of the corresponding characteristic equation, the asymptotic behavior of the solutions and the stability of the trivial solution are explained. Three examples are also provided to illustrate our results.
\end{abstract}

\section{Introduction and Preliminaries}

This paper aims to describe the stability behaviour of the solutions of the neutral-type linear functional differential equations

$$
\frac{d}{d t}\left[x(t)-\int_{-1}^{0} x(t-\tau(\theta)) d q(\theta)\right]=\int_{-1}^{0} x(t-r(\theta)) d v(\theta), \quad t \geq 0
$$

where $x(t) \in \mathbb{R}, r(\theta)$ and $\tau(\theta)$ are nonnegative real continuous functions in $[-1,0]$, and $v(\theta)$ and $q(\theta)$ are real functions of bounded variation in $[-1,0]$. Riemann-Stieltjes integrals are used. It is assumed that $v$ and $q$ are non-constant in $[-1,0]$. Consider the value $R=\max \{\|\tau\|,\|r\|\}$, where $\|\tau\|=\max \{\tau(\theta):-1 \leq \theta \leq 0\}$ and $\|r\|=\max \{r(\theta):-1 \leq \theta \leq 0\}$. The initial condition for (1.1) is determined by a function

$$
x(t)=\phi(t), \quad-R \leq t \leq 0 .
$$

A solution of (1.1) refers to a continuous function $x:[-R,+\infty) \rightarrow \mathbb{R}$ satisfying (1.2), such that

$$
x(t)-\int_{-1}^{0} x(t-\tau(\theta)) d q(\theta)
$$

is differentiable in $[0,+\infty)$ and satisfies (1.1) for every $t \geq 0$.

For a solution of (1.1) in the form $x(t)=e^{\lambda t}$ for $t \in \mathbb{R}, \lambda$ represents a root of the characteristic equation

$$
\lambda\left(1-\int_{-1}^{0} e^{-\lambda \tau(\theta)} d q(\theta)\right)=\int_{-1}^{0} e^{-\lambda r(\theta)} d v(\theta) .
$$

Additionally, this is applied to the relevant class of differential - difference equation

$$
\frac{d}{d t}\left[x(t)-\sum_{j=1}^{m} b_{j} x\left(t-\tau_{j}\right)\right]=\sum_{j=1}^{m} a_{j} x\left(t-r_{j}\right),
$$


where $j=1, \ldots, m, a_{j}, b_{j} \in \mathbb{R}$ and $\tau_{j}, r_{j} \in(0, \infty)$. As it is well-known, this equation can be obtained from (1.1), under the assumption that $q(\theta)$ and $v(\theta)$ are step functions with a number $m$ of jump points (See, [5], [12], [22], [26] and references therein). Furthermore, the equation (1.1) for $\tau(\theta)=r(\theta)=-r \theta \quad(r>0)$ and $\theta \in[-1,0]$ is reduced to the class of the equation

$$
\frac{d}{d t}\left[x(t)-\int_{-1}^{0} x(t+\theta) d q(\theta)\right]=\int_{-1}^{0} x(t+\theta) d v(\theta),
$$

where $q(\theta)=q(\theta / r)$ is atomic at zero, and $v(\theta)=v(\theta / r)$. The authors in [23] and [25] obtained behavior and stability analysis of the solutions of the equation (1.4). In this article, we examine the stability of equation (1.1), which is more general than equation (1.4). In other words, we make preference relying on equation (1.1) considering the chance to understand the impact of the delays on the stability behaviour of the neutral functional differential equations more clearly. One may look at the references [2], [7], [17], [19]- [21], [27] for a special case of equation (1.1)

$$
x^{\prime}(t)=\int_{-1}^{0} x(t-r(\theta)) d v(\theta) .
$$

In addition, the references [4] and [11] may also be reviewed.

Ferreira and Pedro [6] established the oscillatory criteria of the equation (1.1). However, the article in [6] has no information about asymptotic behavior and exponential estimate of solutions. In this article, we obtain the stability analysis of the solutions of the equation (1.1). Namely, we obtained the asymptotic behavior of the solutions and then we created a useful exponential estimate for these solutions and finally provided a stability criterion in this article which is different from the article in [6]. These results are obtained with a real root of the characteristic equation. For this purpose, the applied techniques are generated from a mix of methods used in the references [12], [13], [22]- [25], [27] Examples are also given in this article.

The stability theory of the delay and the differential equations of neutral type in recent two decades has received widespread attention, as one can see through the textbooks [1], [3], [8]- [10], [14], [15], [18] and the references therein. Additionally, there are equations similar to (1.1) in the book by Kolmanovskii and Nosov [9].

Throughout this paper, $V(q)$ and $V(v)$ are denoted for the total variation function of $q$ and $v$, respectively, defined in the interval $[-1,0]$. Note that the functions $V(q)$ and $V(v)$ are greater than zero in the interval $[-1,0]$. Moreover, it must be noted that $V(q)$ and $V(v)$ are not identically zero in the interval $[-1,0]$. The reader should know about both theories of the bounded variation functions and the Riemann-Stieltjes integrations. It is assumed that the reader knows the theory of Riemann-Stieltjes integration and the theory of functions of bounded variation (see [8], [16]).

Finally, in this section, we will give three well-known definitions of stability (see, for example, [9]). The trivial solution of (1.1) is defined as stable if for every $\varepsilon>0$, there exists a number $\ell=\ell(\varepsilon)$ such that, for any initial function $\phi$ with

$$
\|\phi\|=\max _{-R \leq t \leq 0}|\phi(t)|<\ell
$$

the solution $x$ of (1.1)-(1.2) satisfies

$$
|x(t)|<\varepsilon, \quad \text { for all } t \in[-R, \infty) .
$$

In another case, the trivial solution of (1.1) is considered to be unstable. Provided that it is stable in the above-mentioned concept, the trivial solution of (1.1) is also considered asymptotically stable, and additionally, there exists a number $\ell_{0}>0$ such that, for any initial function $\phi$ with $\|\phi\|<\ell_{0}$, the solution $x$ of (1.1)-(1.2) satisfies

$$
\lim _{t \rightarrow \infty} x(t)=0 .
$$

\section{Statement of the Main Results and Comments}

Theorem 2.1. We assume that $\lambda_{0} \in \mathbb{R}$ is a root of characteristic equation (1.3) with the property

$$
\mu\left(\lambda_{0}\right)=\int_{-1}^{0} e^{-\lambda_{0} \tau(\theta)}\left(1+\left|\lambda_{0}\right| \tau(\theta)\right) d V(q)(\theta)+\int_{-1}^{0} e^{-\lambda_{0} r(\theta)} r(\theta) d V(v)(\theta)<1 .
$$

Set

$$
\beta\left(\lambda_{0}\right)=\int_{-1}^{0} e^{-\lambda_{0} \tau(\theta)}\left(\lambda_{0} \tau(\theta)-1\right) d q(\theta)+\int_{-1}^{0} e^{-\lambda_{0} r(\theta)} r(\theta) d v(\theta) .
$$

Then, for any function $\phi \in C([-R, 0], \mathbb{R})$, the solution $x$ of (1.1)-(1.2) satisfies

$$
\lim _{t \rightarrow \infty}\left[e^{-\lambda_{0} t} x(t)\right]=\frac{L\left(\lambda_{0} ; \phi\right)}{1+\beta\left(\lambda_{0}\right)},
$$

where

$$
L\left(\lambda_{0} ; \phi\right)=\phi(0)-\int_{-1}^{0}\left[\phi(-\tau(\theta))-\lambda_{0} e^{-\lambda_{0} \tau(\theta)} \int_{-\tau(\theta)}^{0} e^{-\lambda_{0} u} \phi(u) d u\right] d q(\theta)+\int_{-1}^{0} e^{-\lambda_{0} r(\theta)}\left[\int_{-r(\theta)}^{0} e^{-\lambda_{0} u} \phi(u) d u\right] d v(\theta) .
$$

Note: Property (2.1) guarantees that $1+\beta\left(\lambda_{0}\right)>0$. 
Proof. Property (2.1) implies $0<\mu\left(\lambda_{0}\right)<1$. From (2.2) we obtain

$$
\begin{aligned}
\left|\beta\left(\lambda_{0}\right)\right| & =\left|\lambda_{0} \int_{-1}^{0} e^{-\lambda_{0} \tau(\theta)} \tau(\theta) d q(\theta)-\int_{-1}^{0} e^{-\lambda_{0} \tau(\theta)} d q(\theta)+\int_{-1}^{0} e^{-\lambda_{0} r(\theta)} r(\theta) d v(\theta)\right| \\
& \leq\left|\lambda_{0}\right| \int_{-1}^{0} e^{-\lambda_{0} \tau(\theta)} \tau(\theta) d V(q)(\theta)+\int_{-1}^{0} e^{-\lambda_{0} \tau(\theta)} d V(q)(\theta)+\int_{-1}^{0} e^{-\lambda_{0} r(\theta)} r(\theta) d V(v)(\theta) .
\end{aligned}
$$

In this case, $\left|\beta\left(\lambda_{0}\right)\right| \leq \mu\left(\lambda_{0}\right)$ is satisfied, so $\left|\beta\left(\lambda_{0}\right)\right|<1$. Then $1+\beta\left(\lambda_{0}\right)>0$ is the outcome.

Let us define $y(t)=e^{-\lambda_{0} t} x(t), t \in[-R, \infty)$. Then, by considering that $\lambda_{0}$ is a real root of characteristic equation (1.3), for every $t \geq 0$, we obtain

$$
\begin{aligned}
& {\left[x(t)-\int_{-1}^{0} x(t-\tau(\theta)) d q(\theta)\right]^{\prime}-\int_{-1}^{0} x(t-r(\theta)) d v(\theta)} \\
& =e^{\lambda_{0} t}\left\{\left[y(t)-\int_{-1}^{0} e^{-\lambda_{0} \tau(\theta)} y(t-\tau(\theta)) d q(\theta)\right]^{\prime}+\lambda_{0}\left[y(t)-\int_{-1}^{0} e^{-\lambda_{0} \tau(\theta)} y(t-\tau(\theta)) d q(\theta)\right]-\int_{-1}^{0} e^{-\lambda_{0} r(\theta)} y(t-r(\theta)) d v(\theta)\right\} \\
& =e^{\lambda_{0} t}\left\{\left[y(t)-\int_{-1}^{0} e^{-\lambda_{0} \tau(\theta)} y(t-\tau(\theta)) d q(\theta)\right]^{\prime}+\left[\lambda_{0} \int_{-1}^{0} e^{-\lambda_{0} \tau(\theta)} d q(\theta)+\int_{-1}^{0} e^{-\lambda_{0} r(\theta)} d v(\theta)\right] y(t)\right. \\
& \left.\quad-\lambda_{0} \int_{-1}^{0} e^{-\lambda_{0} \tau(\theta)} y(t-\tau(\theta)) d q(\theta)-\int_{-1}^{0} e^{-\lambda_{0} r(\theta)} y(t-r(\theta)) d v(\theta)\right\} \\
& =e^{\lambda_{0} t}\left\{\left[y(t)-\int_{-1}^{0} e^{-\lambda_{0} \tau(\theta)} y(t-\tau(\theta)) d q(\theta)\right]^{\prime}+\lambda_{0} \int_{-1}^{0} e^{-\lambda_{0} \tau(\theta)}[y(t)-y(t-\tau(\theta))] d q(\theta)+\int_{-1}^{0} e^{-\lambda_{0} r(\theta)}[y(t)-y(t-r(\theta))] d v(\theta)\right\} .
\end{aligned}
$$

Hence, $x$ satisfies (1.1) for all $t \geq 0$, it follows that $y$ satisfies

$$
\left[y(t)-\int_{-1}^{0} e^{-\lambda_{0} \tau(\theta)} y(t-\tau(\theta)) d q(\theta)\right]^{\prime}=-\lambda_{0} \int_{-1}^{0} e^{-\lambda_{0} \tau(\theta)}[y(t)-y(t-\tau(\theta))] d q(\theta)-\int_{-1}^{0} e^{-\lambda_{0} r(\theta)}[y(t)-y(t-r(\theta))] d v(\theta) .
$$

And then, the initial condition (1.2) becomes

$$
y(t)=e^{-\lambda_{0} t} \phi(t), \quad t \in[-R, 0] .
$$

When equation (2.4) is integrated from 0 to $t$, the following equation is obtained

$$
\begin{aligned}
y(t)- & \int_{-1}^{0} e^{-\lambda_{0} \tau(\theta)} y(t-\tau(\theta)) d q(\theta)=y(0)-\int_{-1}^{0} e^{-\lambda_{0} \tau(\theta)} y(-\tau(\theta)) d q(\theta)-\lambda_{0} \int_{-1}^{0} e^{-\lambda_{0} \tau(\theta)}\left(\int_{0}^{t}[y(s)-y(s-\tau(\theta))] d s\right) d q(\theta) \\
& -\int_{-1}^{0} e^{-\lambda_{0} r(\theta)}\left(\int_{0}^{t}[y(s)-y(s-r(\theta))] d s\right) d v(\theta) \\
= & \phi(0)-\int_{-1}^{0} \phi(-\tau(\theta)) d q(\theta)-\lambda_{0} \int_{-1}^{0} e^{-\lambda_{0} \tau(\theta)}\left(\int_{t-\tau(\theta)}^{t} y(u) d u\right) d q(\theta) \\
& -\int_{-1}^{0} e^{-\lambda_{0} r(\theta)}\left(\int_{t-r(\theta)}^{t} y(u) d u\right) d v(\theta)+\lambda_{0} \int_{-1}^{0} e^{-\lambda_{0} \tau(\theta)}\left(\int_{-\tau(\theta)}^{0} y(u) d u\right) d q(\theta)+\int_{-1}^{0} e^{-\lambda_{0} r(\theta)}\left(\int_{-r(\theta)}^{0} y(u) d u\right) d v(\theta) \\
= & -\lambda_{0} \int_{-1}^{0} e^{-\lambda_{0} \tau(\theta)}\left(\int_{t-\tau(\theta)}^{t} y(u) d u\right) d q(\theta)-\int_{-1}^{0} e^{-\lambda_{0} r(\theta)}\left(\int_{t-r(\theta)}^{t} y(u) d u\right) d v(\theta)+L\left(\lambda_{0} ; \phi\right) .
\end{aligned}
$$

Here, $L\left(\lambda_{0} ; \phi\right)$ is given in Theorem 2.1. So that the following equation (2.6) is obtained

$$
\begin{aligned}
y(t) & -\int_{-1}^{0} e^{-\lambda_{0} \tau(\theta)} y(t-\tau(\theta)) d q(\theta)=-\lambda_{0} \int_{-1}^{0} e^{-\lambda_{0} \tau(\theta)}\left(\int_{t-\tau(\theta)}^{t} y(u) d u\right) d q(\theta) \\
& -\int_{-1}^{0} e^{-\lambda_{0} r(\theta)}\left(\int_{t-r(\theta)}^{t} y(u) d u\right) d v(\theta)+L\left(\lambda_{0} ; \phi\right), \quad t \geq 0 .
\end{aligned}
$$

This equation is equivalent to equation (1.1). Now, let us define following expression:

$$
z(t)=y(t)-\frac{L\left(\lambda_{0} ; \phi\right)}{1+\beta\left(\lambda_{0}\right)}, \quad t \geq-R
$$

If this definition applied to equation (2.6), we obtain

$$
\begin{aligned}
z(t) & -\int_{-1}^{0} e^{-\lambda_{0} \tau(\theta)} z(t-\tau(\theta)) d q(\theta)=-\lambda_{0} \int_{-1}^{0} e^{-\lambda_{0} \tau(\theta)}\left(\int_{t-\tau(\theta)}^{t} z(u) d u\right) d q(\theta) \\
& -\int_{-1}^{0} e^{-\lambda_{0} r(\theta)}\left(\int_{t-r(\theta)}^{t} z(u) d u\right) d v(\theta), \quad t \geq 0
\end{aligned}
$$


Moreover, the following expression is obtained when the initial condition (2.5) is obtained

$$
z(t)=e^{-\lambda_{0} t} \phi(t)-\frac{L\left(\lambda_{0} ; \phi\right)}{1+\beta\left(\lambda_{0}\right)}, \quad t \in[-R, 0] .
$$

Here, $\beta\left(\lambda_{0}\right)$ is given by expression (2.2).

From the definitions of $y$ and $z$, we have

$$
\lim _{t \rightarrow \infty} z(t)=0 \text {. }
$$

We will prove the statement (2.9) later. We define,

$$
M\left(\lambda_{0} ; \phi\right)=\max _{t \in[-R, 0]}\left|e^{-\lambda_{0} t} \phi(t)-\frac{L\left(\lambda_{0} ; \phi\right)}{1+\beta\left(\lambda_{0}\right)}\right| .
$$

In this case, from (2.8), the following expression is obtained:

$$
|z(t)| \leq M\left(\lambda_{0} ; \phi\right), \quad-R \leq t \leq 0 .
$$

Now, let us show that the following inequality is satisfied in the interval $[-R, \infty)$

$$
|z(t)| \leq M\left(\lambda_{0} ; \phi\right) .
$$

Consider an arbitrary number $\varepsilon>0$. We claim that

$$
|z(t)|<M\left(\lambda_{0} ; \phi\right)+\varepsilon, \quad \text { for } t \geq-R .
$$

Let us assume that inequality (2.13) is not satisfied. In this case, because of (2.11), there exist a point $t_{0}>0$ such that

$$
|z(t)|<M\left(\lambda_{0} ; \phi\right)+\varepsilon, \quad-R \leq t<t_{0} \quad \text { and } \quad\left|z\left(t_{0}\right)\right|=M\left(\lambda_{0} ; \phi\right)+\varepsilon
$$

Since $\mu\left(\lambda_{0}\right)<1$, from equation (2.7) we obtain

$$
\begin{aligned}
M\left(\lambda_{0} ; \phi\right)+\varepsilon= & \left|z\left(t_{0}\right)\right|=\mid \int_{-1}^{0} e^{-\lambda_{0} \tau(\theta)} z\left(t_{0}-\tau(\theta)\right) d q(\theta)-\lambda_{0} \int_{-1}^{0} e^{-\lambda_{0} \tau(\theta)}\left(\int_{t_{0}-\tau(\theta)}^{t_{0}} z(u) d u\right) d q(\theta) \\
& -\int_{-1}^{0} e^{-\lambda_{0} r(\theta)}\left(\int_{t_{0}-r(\theta)}^{t_{0}} z(u) d u\right) d v(\theta) \mid \\
\leq & \left|\int_{-1}^{0} e^{-\lambda_{0} \tau(\theta)} z\left(t_{0}-\tau(\theta)\right) d q(\theta)\right|+\left|\lambda_{0}\right|\left|\int_{-1}^{0} e^{-\lambda_{0} \tau(\theta)}\left(\int_{t_{0}-\tau(\theta)}^{t_{0}} z(u) d u\right) d q(\theta)\right| \\
& +\left|\int_{-1}^{0} e^{-\lambda_{0} r(\theta)}\left(\int_{t_{0}-r(\theta)}^{t_{0}} z(u) d u\right) d v(\theta)\right| \\
\leq & \int_{-1}^{0} e^{-\lambda_{0} \tau(\theta)}\left|z\left(t_{0}-\tau(\theta)\right)\right| d V(q)(\theta)+\left|\lambda_{0}\right| \int_{-1}^{0} e^{-\lambda_{0} \tau(\theta)}\left(\int_{t_{0}-\tau(\theta)}^{t_{0}}|z(u)| d u\right) d V(q)(\theta) \\
& +\int_{-1}^{0} e^{-\lambda_{0} r(\theta)}\left(\int_{t_{0}-r(\theta)}^{t_{0}}|z(u)| d u\right) d V(v)(\theta) \\
\leq & {\left[M\left(\lambda_{0} ; \phi\right)+\varepsilon\right]\left\{\int_{-1}^{0} e^{-\lambda_{0} \tau(\theta)} d V(q)(\theta)+\left|\lambda_{0}\right| \int_{-1}^{0} e^{-\lambda_{0} \tau(\theta)} \tau(\theta) d V(q)(\theta)+\int_{-1}^{0} e^{-\lambda_{0} r(\theta)} r(\theta) d V(v)(\theta)\right\} } \\
= & {\left[M\left(\lambda_{0} ; \phi\right)+\varepsilon\right]\left\{\int_{-1}^{0} e^{-\lambda_{0} \tau(\theta)}\left(1+\left|\lambda_{0}\right| \tau(\theta)\right) d V(q)(\theta)+\int_{-1}^{0} e^{-\lambda_{0} r(\theta)} r(\theta) d V(v)(\theta)\right\} } \\
= & {\left[M\left(\lambda_{0} ; \phi\right)+\varepsilon\right] \mu\left(\lambda_{0}\right)<\left[M\left(\lambda_{0} ; \phi\right)+\varepsilon\right], }
\end{aligned}
$$

which is a contradiction. So, inequality (2.13) must be true. Therefore, inequality (2.12) must also be true. Now, by virtue of (2.12), from (2.7) we obtain for $t \geq 0$

$$
\begin{aligned}
|z(t)| & \leq\left|\int_{-1}^{0} e^{-\lambda_{0} \tau(\theta)} z(t-\tau(\theta)) d q(\theta)\right|+\left|\lambda_{0}\right|\left|\int_{-1}^{0} e^{-\lambda_{0} \tau(\theta)}\left(\int_{t-\tau(\theta)}^{t} z(u) d u\right) d q(\theta)\right|+\left|\int_{-1}^{0} e^{-\lambda_{0} r(\theta)}\left(\int_{t-r(\theta)}^{t} z(u) d u\right) d v(\theta)\right| \\
& \leq \int_{-1}^{0} e^{-\lambda_{0} \tau(\theta)}|z(t-\tau(\theta))| d V(q)(\theta)+\left|\lambda_{0}\right| \int_{-1}^{0} e^{-\lambda_{0} \tau(\theta)}\left(\int_{t-\tau(\theta)}^{t}|z(u)| d u\right) d V(q)(\theta)+\int_{-1}^{0} e^{-\lambda_{0} r(\theta)}\left(\int_{t-r(\theta)}^{t}|z(u)| d u\right) d V(v)(\theta) \\
& \leq M\left(\lambda_{0} ; \phi\right)\left\{\int_{-1}^{0} e^{-\lambda_{0} \tau(\theta)} d V(q)(\theta)+\left|\lambda_{0}\right| \int_{-1}^{0} e^{-\lambda_{0} \tau(\theta)} \tau(\theta) d V(q)(\theta)+\int_{-1}^{0} e^{-\lambda_{0} r(\theta)} r(\theta) d V(v)(\theta)\right\} \\
& =M\left(\lambda_{0} ; \phi\right)\left\{\int_{-1}^{0} e^{-\lambda_{0} \tau(\theta)}\left(1+\left|\lambda_{0}\right| \tau(\theta)\right) d V(q)(\theta)+\int_{-1}^{0} e^{-\lambda_{0} r(\theta)} r(\theta) d V(v)(\theta)\right\} .
\end{aligned}
$$


Due to the definition of $\mu\left(\lambda_{0}\right)$, we have

$$
|z(t)| \leq \mu\left(\lambda_{0}\right) M\left(\lambda_{0} ; \phi\right), \quad \forall t \geq 0 .
$$

Using inequalities (2.12) and (2.14), by the induction method we can easily show

$$
|z(t)| \leq\left(\mu\left(\lambda_{0}\right)\right)^{n} M\left(\lambda_{0} ; \phi\right), \quad t \geq n R-R \quad(n=0,1,2, \ldots) .
$$

Here, due to $\lim _{n \rightarrow \infty}\left(\mu\left(\lambda_{0}\right)\right)^{n}=0$, from inequality (2.15) $\lim _{t \rightarrow \infty} z(t)=0$ is obtained, that is, (2.9) is true. Hence, Theorem 2.1 is proven at all.

A root of characteristic equation (1.3) is $\lambda=0$ if and only if the following expressions hold:

$$
\int_{-1}^{0} d v(\theta)=0 \quad \text { and } \quad \int_{-1}^{0} d V(q)(\theta)+\int_{-1}^{0} r(\theta) d V(v)(\theta)<1
$$

or

$$
v(0)=v(-1) \quad \text { and } \quad V(q)(0)-V(q)(-1)+\int_{-1}^{0} r(\theta) d V(v)(\theta)<1 .
$$

So, an application of Theorem 2.1 with $\lambda=0$ leads to the following corollary.

Corollary 2.2. Let us satisfy the conditions of (2.16). In this case, for any $\phi \in C([-R, 0], \mathbb{R})$, the solution $x$ of equation (1.1)-(1.2) is given as follows:

$$
\lim _{t \rightarrow \infty} x(t)=\frac{\phi(0)-\int_{-1}^{0} \phi(-\tau(\theta)) d q(\theta)+\int_{-1}^{0}\left[\int_{-r(\theta)}^{0} \phi(u) d u\right] d v(\theta)}{1-q(0)+q(-1)+\int_{-1}^{0} r(\theta) d v(\theta)} .
$$

Note: Because of the second condition of (2.16), $1-q(0)+q(-1)+\int_{-1}^{0} r(\theta) d v(\theta)>0$ holds.

Theorem 2.3. Let $\lambda_{0}$ be a real root of the characteristic equation (1.3), and the condition (2.1) is provided for $\lambda_{0}$. Let us consider $\beta\left(\lambda_{0}\right)$ in Theorem 2.1. Then, for any $\phi \in C([-R, 0], \mathbb{R})$, the solution $x$ of (1.1)-(1.2) satisfies

$$
|x(t)| \leq\left[\frac{\left(1+\mu\left(\lambda_{0}\right)\right)^{2}}{1+\beta\left(\lambda_{0}\right)}+\mu\left(\lambda_{0}\right)\right] N\left(\lambda_{0} ; \phi\right) e^{\lambda_{0} t}, \quad \forall t \geq 0
$$

where

$$
N\left(\lambda_{0} ; \phi\right)=\max _{t \in[-R, 0]}\left|e^{-\lambda_{0} t} \phi(t)\right| .
$$

Moreover, the trivial solution of equation (1.1) is stable if $\lambda=0$, asymptotically stable if $\lambda_{0}<0$ and unstable if $\lambda_{0}>0$.

Proof. Let $y$ and $z$ be defined as in the proof of Theorem 2.1, i.e.

$$
z(t)=y(t)-\frac{L\left(\lambda_{0} ; \phi\right)}{1+\beta\left(\lambda_{0}\right)}, \quad t \geq-R
$$

where $\left.L\left(\lambda_{0} ; \phi\right)\right)$ and $M\left(\lambda_{0} ; \phi\right)$ are defined as in Theorem 2.1. Then, we can express the following for $t \geq 0$

$$
y(t) \leq \frac{\left|L\left(\lambda_{0} ; \phi\right)\right|}{1+\beta\left(\lambda_{0}\right)}+\mu\left(\lambda_{0}\right) M\left(\lambda_{0} ; \phi\right) .
$$

From the definition of $L\left(\lambda_{0} ; \phi\right)$ we get

$$
\begin{aligned}
L\left(\lambda_{0} ; \phi\right)= & \phi(0)-\int_{-1}^{0}\left[e^{\lambda_{0} \tau(\theta)} \phi(-\tau(\theta))-\lambda_{0} \int_{-\tau(\theta)}^{0} e^{-\lambda_{0} u} \phi(u) d u\right] e^{-\lambda_{0} \tau(\theta)} d q(\theta)+\int_{-1}^{0} e^{-\lambda_{0} r(\theta)}\left[\int_{-r(\theta)}^{0} e^{-\lambda_{0} u} \phi(u) d u\right] d v(\theta) \\
\leq & |\phi(0)|+\int_{-1}^{0}\left[\left|e^{\lambda_{0} \tau(\theta)} \phi(-\tau(\theta))\right|+\left|\lambda_{0}\right| \int_{-\tau(\theta)}^{0}\left|e^{-\lambda_{0} u} \phi(u)\right| d u\right] e^{-\lambda_{0} \tau(\theta)} d V(q)(\theta) \\
& +\int_{-1}^{0} e^{-\lambda_{0} r(\theta)}\left[\int_{-r(\theta)}^{0}\left|e^{-\lambda_{0} u} \phi(u)\right| d u\right] d V(v)(\theta) \\
\leq & \left\{1+\int_{-1}^{0}\left[1+\left|\lambda_{0}\right| \tau(\theta)\right] e^{-\lambda_{0} \tau(\theta)} d V(q)(\theta)+\int_{-1}^{0} e^{-\lambda_{0} r(\theta)} r(\theta) d V(v)(\theta)\right\} N\left(\lambda_{0} ; \phi\right) \\
= & \left(1+\mu\left(\lambda_{0}\right)\right) N\left(\lambda_{0} ; \phi\right) .
\end{aligned}
$$

Furthermore, by the definition of $\mu\left(\lambda_{0} ; \phi\right)$, we obtain

$$
\begin{aligned}
M\left(\lambda_{0} ; \phi\right) & =\max _{t \in[-R, 0]}\left|e^{-\lambda_{0} t} \phi(t)-\frac{L\left(\lambda_{0} ; \phi\right)}{1+\beta\left(\lambda_{0}\right)}\right| \leq \max _{t \in[-R, 0]}\left|e^{-\lambda_{0} t} \phi(t)\right|+\frac{\left|L\left(\lambda_{0} ; \phi\right)\right|}{1+\beta\left(\lambda_{0}\right)} \\
& =N\left(\lambda_{0} ; \phi\right)+\frac{\left|L\left(\lambda_{0} ; \phi\right)\right|}{1+\beta\left(\lambda_{0}\right)} \leq N\left(\lambda_{0} ; \phi\right)+\frac{\left(1+\mu\left(\lambda_{0}\right)\right) N\left(\lambda_{0} ; \phi\right)}{1+\beta\left(\lambda_{0}\right)} \\
& =\left(1+\frac{1+\mu\left(\lambda_{0}\right)}{1+\beta\left(\lambda_{0}\right)}\right) N\left(\lambda_{0} ; \phi\right) .
\end{aligned}
$$


So, from (2.18) we get

$$
\begin{aligned}
|y(t)| & \leq \frac{\left(1+\mu\left(\lambda_{0}\right)\right) N\left(\lambda_{0} ; \phi\right)}{1+\beta\left(\lambda_{0}\right)}+\mu\left(\lambda_{0}\right)\left(1+\frac{1+\mu\left(\lambda_{0}\right)}{1+\beta\left(\lambda_{0}\right)}\right) N\left(\lambda_{0} ; \phi\right) \\
& =\left[\frac{\left(1+\mu\left(\lambda_{0}\right)\right)^{2}}{1+\beta\left(\lambda_{0}\right)}+\mu\left(\lambda_{0}\right)\right] N\left(\lambda_{0} ; \phi\right) .
\end{aligned}
$$

Finally, by the definition of $y$, we obtain

$$
|x(t)| \leq\left[\frac{\left(1+\mu\left(\lambda_{0}\right)\right)^{2}}{1+\beta\left(\lambda_{0}\right)}+\mu\left(\lambda_{0}\right)\right] N\left(\lambda_{0} ; \phi\right) e^{\lambda_{0} t}
$$

for all $t \geq 0$. So, the proof of the first part of the theorem is completed. Now let us show the stability criterion of the theorem.

Assume that $\lambda_{0} \leq 0$, and let $\phi \in([-R, 0], \mathbb{R})$ be an arbitrary initial function. Then, from inequality (2.19) we get

$$
|x(t)| \leq\left[\frac{\left(1+\mu\left(\lambda_{0}\right)\right)^{2}}{1+\beta\left(\lambda_{0}\right)}+\mu\left(\lambda_{0}\right)\right] N\left(\lambda_{0} ; \phi\right), \quad \forall t \geq 0 .
$$

Because $\frac{\left(1+\mu\left(\lambda_{0}\right)\right)^{2}}{1+\beta\left(\lambda_{0}\right)}>1$, we obtain

$$
|x(t)| \leq\left[\frac{\left(1+\mu\left(\lambda_{0}\right)\right)^{2}}{1+\beta\left(\lambda_{0}\right)}+\mu\left(\lambda_{0}\right)\right] N\left(\lambda_{0} ; \phi\right), \quad \forall t \geq-R .
$$

So, the trivial solution of equation (1.1) is stable. For $\lambda_{0}<0$, it is clear that, from inequality (2.19) it follows that

$$
\lim _{t \rightarrow \infty} x(t)=0
$$

Thus, the trivial solution is asymptotically stable. Finally, let $\lambda_{0}>0$. We want to show that this solution is unstable. Let us assume that it is stable. Then, there is a number $\delta>0$ such that, for each $\phi \in C([-R, 0], \mathbb{R})$ with $\|\phi\|<\delta$, the solution $x$ of (1.1)-(1.2) satisfies

$$
|x(t)|<1, \quad t \geq-R
$$

Given the following,

$$
\phi_{0}(t)=e^{\lambda_{0} t}, \quad t \in[-R, 0]
$$

We see that $\phi_{0} \in C([-R, 0], \mathbb{R})$. From the definition of $L\left(\lambda_{0} ; \phi\right)$ we obtain

$$
\begin{aligned}
L\left(\lambda_{0} ; \phi_{0}\right) & \equiv \phi_{0}(0)-\int_{-1}^{0}\left[\phi_{0}(-\tau(\theta))-\lambda_{0} e^{-\lambda_{0} \tau(\theta)} \int_{-\tau(\theta)}^{0} e^{-\lambda_{0} u} \phi_{0}(u) d u\right] d q(\theta)+\int_{-1}^{0} e^{-\lambda_{0} r(\theta)}\left[\int_{-r(\theta)}^{0} e^{-\lambda_{0} u} \phi_{0}(u) d u\right] d v(\theta) \\
& =1-\int_{-1}^{0}\left[e^{-\lambda_{0} \tau(\theta)}-\lambda_{0} e^{-\lambda_{0} \tau(\theta)} \int_{-\tau(\theta)}^{0} e^{-\lambda_{0} u} e^{\lambda_{0} u} d u\right] d q(\theta)+\int_{-1}^{0} e^{-\lambda_{0} r(\theta)}\left[\int_{-r(\theta)}^{0} e^{-\lambda_{0} u} e^{\lambda_{0} u} d u\right] d v(\theta) \\
& =1-\int_{-1}^{0} e^{-\lambda_{0} \tau(\theta)}\left[1-\lambda_{0} \tau(\theta)\right] d q(\theta)+\int_{-1}^{0} e^{-\lambda_{0} r(\theta)} r(\theta) d v(\theta) \\
& =1+\beta\left(\lambda_{0}\right)>0 .
\end{aligned}
$$

Now, we select a number $\delta_{0}$ with $0<\delta_{0}<\delta$ and let

$$
\phi=\frac{\delta_{0}}{\left\|\phi_{0}\right\|} \phi_{0}
$$

where it is clear that $\phi \in C([-R, 0], \mathbb{R})$ and $\|\phi\|=\delta_{0}<\delta$. So, we obtain

$$
\lim _{t \rightarrow \infty}\left[e^{-\lambda_{0} t} x(t)\right]=\frac{L\left(\lambda_{0} ; \phi\right)}{1+\beta\left(\lambda_{0}\right)}=\frac{\left(\delta_{0} /\left\|\phi_{0}\right\|\right) L\left(\lambda_{0} ; \phi_{0}\right)}{1+\beta\left(\lambda_{0}\right)}=\frac{\delta_{0}}{\left\|\phi_{0}\right\|}>0
$$

However, due to $\lambda_{0}>0$, we get

$$
\lim _{t \rightarrow \infty}\left[e^{-\lambda_{0} t} x(t)\right]=0 .
$$

We reach a contradiction, and hence Theorem 2.3 is proven. 


\section{Examples}

Example 3.1. Let $\tau(\theta)=-\frac{\theta}{4}, r(\theta)=\frac{\theta^{2}}{2}, q(\theta)=-\frac{\theta^{2}}{5}$ and $v(\theta)=\frac{3 \theta^{2}}{5}$. In this case, the characteristic equation (1.3) is obtained as follows:

$$
\lambda\left(1-\int_{-1}^{0} \exp \left(\frac{\lambda \theta}{4}\right) d\left(-\frac{\theta^{2}}{5}\right)\right)=\int_{-1}^{0} \exp \left(-\frac{\lambda \theta^{2}}{2}\right) d\left(\frac{3 \theta^{2}}{5}\right) .
$$

It is seen that $\lambda_{0} \cong-1,03$ is a root of equation (3.1). Here, this root is one of the roots of (3.1) that we obtain with the MATLAB program. Thus, the condition of Theorem 2.3 is satisfied by using the root $\lambda_{0}=-1,03$. Namely, because the functions $q$ and $v$ are monotonous in $[-1,0]$, from expression (2.1) we obtain

$$
\begin{aligned}
\mu(-1,03) & =\int_{-1}^{0} e^{-\frac{1,03 \theta}{4}}\left(1+|-1,03|\left(-\frac{\theta}{4}\right)\right) d V\left(-\frac{\theta^{2}}{5}\right)+\int_{-1}^{0} e^{\frac{1,03 \theta^{2}}{2}}\left(\frac{\theta^{2}}{2}\right) d V\left(\frac{3 \theta^{2}}{5}\right) \\
& \leq \max _{-1 \leq \theta \leq 0}\left|e^{-\frac{1,03 \theta}{4}}\left(1-\frac{1,03 \theta}{4}\right)\right|\left|-\frac{1}{5}\right|+\max _{-1 \leq \theta \leq 0}\left|e^{\frac{1,03 \theta^{2}}{2}}\left(\frac{\theta^{2}}{2}\right)\right|\left|\frac{3}{5}\right| \\
& =\frac{1}{5} e^{\frac{1,03}{4}}\left(1+\frac{1,03}{4}\right)+\frac{3}{5} \frac{e^{\frac{1,03}{2}}}{2} \cong 0,83<1 .
\end{aligned}
$$

Since $\lambda_{0}=-1,03<0$, the solution $x(t) \equiv 0$ is asymptotically stable.

Example 3.2. Let $\tau(\theta)=\theta^{2}, r(\theta)=-\frac{\theta}{2}, q(\theta)=-\frac{\theta^{2}}{4}$ and $v(\theta)=4 \theta^{3}$. In this case, the characteristic equation (1.3) is obtained as follows:

$$
\lambda\left(1-\int_{-1}^{0} \exp \left(-\lambda \theta^{2}\right) d\left(-\frac{\theta^{2}}{4}\right)\right)=\int_{-1}^{0} \exp \left(\frac{\lambda \theta}{2}\right) d\left(4 \theta^{3}\right) .
$$

We see $\lambda_{0} \cong 2,08745$ is a root of equation (3.2). Here, this root is one of the roots of (3.2) that we obtain with the MATLAB program. Thus, the condition of Theorem 2.3 is satisfied for the root. Namely, because the functions $q$ and $v$ are monotonous in $[-1,0]$, from (2.1) we obtain

$$
\begin{aligned}
\mu(2,08745) & =\int_{-1}^{0} e^{-2,08745 \theta^{2}}\left(1+2,08745 \theta^{2}\right) d V\left(-\frac{\theta^{2}}{4}\right)+\int_{-1}^{0} e^{\frac{2,08745 \theta}{2}}\left(-\frac{\theta}{2}\right) d V\left(4 \theta^{3}\right) \\
& \leq \max _{-1 \leq \theta \leq 0}\left|e^{-2,08745 \theta^{2}}\left(1+2,08745 \theta^{2}\right)\right| \frac{1}{4}+\max _{-1 \leq \theta \leq 0}\left|e^{\frac{2,08745 \theta}{2}}\left(-\frac{\theta}{2}\right)\right| 4 \\
& =e^{0}(1+0) \frac{1}{4}+\frac{e^{-\frac{2,08745}{2}}}{2} 4=0,954<1 .
\end{aligned}
$$

Since $\lambda_{0}>0$, the solution $x(t) \equiv 0$ is unstable.

Example 3.3. Let $\tau(\theta)=-2 \theta, r(\theta)=-\frac{\theta}{2}, v(\theta)=\frac{\theta^{2}+\theta}{2}$ and $q(\theta)=\frac{\theta}{4}$. In this case, the characteristic equation (1.3) is obtained as follows:

$$
\lambda\left(1-\int_{-1}^{0} \exp (2 \lambda \theta) d\left(\frac{\theta}{4}\right)\right)=\int_{-1}^{0} \exp \left(\frac{\lambda \theta}{2}\right) d\left(\frac{\theta^{2}+\theta}{2}\right) .
$$

We see $\lambda_{0}=0$ is a root of equation (3.3). The following expressions is satisfied since the function $v(\theta)$ is decreasing in the interval $\left[-1,-\frac{1}{2}\right]$ and increasing in the interval $\left[-\frac{1}{2}, 0\right]$, whereas $q(\theta)$ is also increasing in the interval $[-1,0]$ :

$$
\begin{aligned}
\mu(0) & =\int_{-1}^{0} d V\left(\frac{\theta}{4}\right)+\int_{-1}^{0}\left(-\frac{\theta}{2}\right) d V\left(\frac{\theta^{2}+\theta}{2}\right) \\
& =\int_{-1}^{0} \frac{1}{4} d V(\theta)+\int_{-1}^{-\frac{1}{2}}\left(-\frac{\theta}{2}\right) d V\left(\frac{\theta^{2}+\theta}{2}\right)+\int_{-\frac{1}{2}}^{0}\left(-\frac{\theta}{2}\right) d V\left(\frac{\theta^{2}+\theta}{2}\right) \\
& \leq \frac{1}{4}+\max _{-1 \leq \theta \leq 0}\left\{\left(-\frac{\theta}{2}\right)\right\} \frac{1}{2}\left[\int_{-1}^{-\frac{1}{2}} d V\left(\theta^{2}+\theta\right)+\int_{-\frac{1}{2}}^{0} d V\left(\theta^{2}+\theta\right)\right] \\
& =\frac{1}{4}+\frac{1}{4}\left(\left|-\frac{1}{4}\right|+\left|\frac{1}{4}\right|\right)=\frac{1}{4}+\frac{1}{4} \frac{1}{2}=\frac{3}{8} \\
& <1 .
\end{aligned}
$$

Hence, the solution $x(t) \equiv 0$ is stable for the root $\lambda_{0}=0$. 


\section{Conclusions}

In this study, firstly, a basic asymptotic result for the solution of the equation (1.1) is proved. Secondly, we obtained a useful exponential boundary for solutions and the stability of trivial solutions were shown. These results were obtained using a suitable real root for the characteristic equation. Namely, this real root played an important role in establishing the results of the article. Finally, three examples were given for stability.

\section{References}

[1] T.A. Burton, Volterra integral and differential equations, Academic Press, New York, 1983.

[2] K.L. Cooke, J.M. Ferreira, Stability conditions for linear retarded functional differential equations, J. Math. Anal. Appl., 96 (1983), $480-504$.

[3] C. Corduneanu, Integral equations and applications, Cambridge University Press, New York, 1991.

[4] J.M. Ferreira, I. Györİ, Oscillatory behavior in linear retarded functional differential equations, J. Math. Anal. Appl., 128 (1987), $332-346$.

[5] J.M. Ferreira, A.M. Pedro, Oscillations of differential-difference systems of neutral type, J. Math. Anal. Appl., 253 (2001), $274-289$.

[6] J.M. Ferreira, A.M. Pedro, Oscillatory behaviour in functional differential systems of neutral type, J. Math. Anal. Appl., 269 (2002), $533-555$.

[7] N.J. Ford, Y. Yan, Md.A. Malique, Numerical treatment of oscillatory functional differential equations, J. of Comput. and Appl. Math., 234 (2010), $2757-2767$

[8] J.K. Hale, S.M. Verduyn Lunel, Introduction to functional differential equations, Springer, Berlin, Heidelberg, New York, 1993.

[9] V.B. Kolmanovskii, V.R. Nosov, Stability of functional differential equations, Academic Press, Inc., London, 1986.

[10] V.B. Kolmanovskii, A. Myshkis, Applied theory of functional differential equations, Kluver Academic, Dordrecht, 1992.

[11] Q. Kong, Oscillation for systems of functional differential equations, J. Math. Anal. Appl., 198 (1996), 608-619.

[12] I.G. E. Kordonis, N. T. Niyianni, Ch. G. Philos, On the behavior of the solutions of scalar first order linear autonomous neutral delay differential equations, Arch. Math. (Basel), 71 (1998), 454-464.

[13] I.G.E. Kordonis, Ch.G. Philos, The behavior of solutions of linear integro-differential equations with unbounded delay, Comput. Math. Appl., 38 (1999), $45-50$

[14] Y. Kuang, Delay differential equations with applications in population dynamics, Academic Press, San Diego, 1993.

[15] V. Lakshmikantham, L. Wen, B. Zhang, Theory of differential equations with unbounded delay, Kluwer Academic Publishers, London, 1994.

[16] K. Leffler, The Riemann-Stieltjes integral and some applications in complex analysis and probability theory. Umea University, Institutionen för matematik och matematisk statistik, 2014.

[17] Md. A. Malique, Numerical treatment of oscillatory delay and mixed functional differential equations arising in modeling, PhD thesis, University of Liverpool (University of Chester), 2012

[18] S.I. Niculescu, Delay effects on stability, Springer-Verlag London Limited, 2001.

[19] A.M. Pedro, Oscillation and nonoscillation criteria for retarded functional differential equations, in: International Conference on Differential Equations (Proc. Internat. Sympos., Czecho-Slovak series, Bratislava, 2005), Comenius University Press, Bratislava, (2007), 353-362.

[20] A.M. Pedro, Nonoscillation criteria for retarded functional differential systems, Inter. J. of Pure and Appl. Math., 31 (2006), 47-71.

[21] A.M. Pedro, Oscillation criteria for retarded functional differential systems, Inter. J. of Pure and Appl. Math., 63 (2010), $75-84$.

[22] Ch.G. Philos, I.K. Purnaras, Periodic first order linear neutral delay differential equations, Appl. Math. Comput., 117 (2001), $203-222$.

[23] Ch.G. Philos, I.K. Purnaras, Asymptotic properties, nonoscillation, and stability for scalar first order linear autonomous neutral delay differential equations, Electron. J. Differential Equations, 03 (2004), 1-17.

[24] Ch.G. Philos, I.K. Purnaras, More on the behavior of solutions to linear integro differantial equations with unbounded delay, Funkcial. Ekvac., 48 (2005), 393-414.

[25] Ch.G. Philos, I.K. Purnaras, A result on the behavior of the solutions for scalar first order linear autonomous neutral delay differential equations, Math. Proc. Camb. Phil. Soc., 140 (2006), 349-358.

[26] U. Stroinski, Order and oscillation in delay differential systems, J. Math. Anal. Appl., 207 (1997), $158-171$.

[27] A.F. Yeniçerioglu, C. Yazıc1, Stability criteria for retarded functional differential equations. Eskişehir Technical Univ. J. of Sci. and Tech. B - Theo.Sci., $\mathbf{8}(2),(2020), 212-222$ 